\title{
Reflets
}

Revue ontaroise d'intervention sociale et communautaire

\section{Le développement des services en français en matière de violence faite aux femmes : des pratiques à notre image}

\section{Ghislaine Sirois et Marie-Luce Garceau}

Volume 13, numéro 1, 2007

La violence dans tous ses états

URI : https://id.erudit.org/iderudit/016814ar

DOI : https://doi.org/10.7202/016814ar

Aller au sommaire du numéro

Éditeur(s)

Reflets : Revue ontaroise d'intervention sociale et communautaire

ISSN

1203-4576 (imprimé)

1712-8498 (numérique)

Découvrir la revue

Citer cet article

Sirois, G. \& Garceau, M.-L. (2007). Le développement des services en français en matière de violence faite aux femmes : des pratiques à notre image. Reflets, 13(1), 98-111. https://doi.org/10.7202/016814ar

Tous droits réservés (C) Reflets : Revue ontaroise d'intervention sociale et communautaire, 2007
Ce document est protégé par la loi sur le droit d'auteur. L'utilisation des services d’Érudit (y compris la reproduction) est assujettie à sa politique d'utilisation que vous pouvez consulter en ligne.

https://apropos.erudit.org/fr/usagers/politique-dutilisation/ 


\title{
Le développement des services en français en matière de violence faite aux femmes : des pratiques à notre image
}

\author{
Ghislaine Sirois, \\ Action ontarienne contre la violence faite aux femmes \\ (Ottawa) \\ et \\ Marie-Luce Garceau, \\ École de service social, Université Laurentienne
}

\section{Introduction}

Cet article porte sur ce qu'Action ontarienne contre la violence faite aux femmes $(\mathrm{AOcVF})$ et ses groupes membres considèrent comme leurs meilleures pratiques entourant le développement des services en français (SEF) dans le domaine de la violence faite aux femmes. Nous présentons d'abord AOcVF. Puis, nous discutons brièvement des droits des francophones en Ontario et de ce que nous considérons comme un service en français de qualité. Nous présentons enfin nos "meilleures pratiques". Vous constaterez à leur lecture qu'elles ont été développées pour répondre aux besoins des femmes francophones disséminées à la grandeur de la province. Vous comprendrez également qu'en matière de violence faite aux femmes, les services en français sont offerts par un nombre limité d'organismes disposant le plus souvent de budgets restreints. 
"Pour AOcVF et ses membres, l'offre de SEF aux femmes francophones aux prises avec de la violence est un droit acquis et chèrement défendu. »

\section{Action ontarienne contre la violence faite aux femmes}

AOcVF a vu le jour en 1988. Il s'agit d'un organisme provincial regroupant les maisons d'hébergement pour femmes victimes de violence, les centres d'aide et de lutte contre les agressions à caractère sexuel (Calacs) et quelques autres organismes offrant des SEF en matière de violence faite aux femmes. L'AOcVF veut prévenir la violence faite aux femmes par :

- la concertation et le démarchage visant la mise sur pied de SEF pour les femmes victimes de violence;

- la formation comme soutien pour les intervenantes;

- la création de matériel en français (documents à l'intention des usagères, analyses d'enjeux, etc.).

Pour AOcVF et ses membres, l'offre de SEF aux femmes francophones aux prises avec de la violence est un droit acquis et chèrement défendu.

\section{Les droits acquis sur le plan des services en français}

Une fois pour toutes, mettons un terme au mythe voulant que la demande de services en français soit "un caprice " ou une " toquade » de la population d'expression française, ou encore que les SEF dédoublent des services déjà existants. Ce mythe perdure et est tout particulièrement dommageable quand il s'agit d'offrir des services aux femmes francophones violentées.

Premièrement, les femmes francophones ne sont pas toutes bilingues. Elles ne sont pas toutes capables de faire valoir leurs besoins dans la langue de la majorité. Et si on pense aux femmes de la récente vague d'immigration francophone, surtout africaine, attirées ici par la publicité qui présente le Canada comme «le plus meilleur pays bilingue au monde »-pour paraphraser les affirmations de notre ex premier ministre - beaucoup parmi elles n'ont pas accès aux services qui les mettraient à l'abri de la violence, essentiellement parce qu'elles sont d'expression française. 
"Il va sans dire que malgré ses initiatives le gouvernement de l'Ontario ne respecte pas encore toutes ses obligations dans le domaine de la violence contre les femmes. "
Deuxièmement, il existe en Ontario des droits acquis quant aux services en français, entre autres :

- Les garanties de la Constitution canadienne visant le respect et la protection des minorités linguistiques de langues officielles au Canada, donc de la minorité linguistique francophone en Ontario et dans les autres provinces, sauf au Québec où ce sont les droits de la minorité linguistique d'expression anglaise qui sont protégés;

- La Loi de 1986 sur les services en français, entrée en vigueur en 1989, reconnaissant le rôle historique des francophones. De plus, elle définit les obligations du gouvernement de l'Ontario en matière de services en français. Selon cette loi, et selon l'interprétation donnée par la décision rendue en faveur du maintien de l'Hôpital Montfort à Ottawa, les francophones ont droit à des services en français de qualité, dans le but de protéger la communauté linguistique francophone et de favoriser l'égalité entre le français et l'anglais en Ontario.

Il va sans dire que malgré ses initiatives le gouvernement de l'Ontario ne respecte pas encore toutes ses obligations dans le domaine de la violence contre les femmes.

\section{Les services en français de qualité en matière de violence}

Malgré les lacunes dans l'offre de SEF, AOcVF et ses membres ont établi les éléments définissant de façon concrète ce que sont des SEF de qualité dans le domaine de la violence. En voici les principaux éléments :

1. À tous les niveaux de service, le personnel est francophone et compétent. Il peut s'exprimer couramment en français et il est sensible à l'oppression des francophones en milieu minoritaire et à leur diversité;

2. Les ressources humaines et financières sont suffisantes pour offrir des SEF de façon continue;

3. Tous les SEF offerts sont garantis et accessibles en tout temps. Toute la gamme des services est disponible en français et offerte 
"Ce sont des maisons d'hébergement qui offrent encore aujourd'hui des SEF en tout temps, avec quelques travailleuses en counselling dans des postes satellites multiservices." en permanence. Le matériel en français est facile à comprendre et à utiliser pour toutes les femmes;

4. Les services ont une approche féministe et holistique respectant la diversité des femmes;

5. La structure organisationnelle appuie l'offre et la création de services par et pour les femmes francophones dans leur diversité.

Bref, ce sont ces éléments qui sont au cœur des SEF en matière de violence faite aux femmes. Toutefois, plusieurs obstacles nuisent à leur mise en application. Afin de les surmonter, $\mathrm{AOcVF}$ et ses membres ont instauré, au cours des années, ce que nous appelons nos "meilleures pratiques». Elles vont de pair avec la création des services et les vagues d'investissements des gouvernements successifs.

\section{La meilleure pratique numéro un : des services en français autonomes}

La création de services en violence contre les femmes a commencé au début des années 1970. À cette époque et tout au long des années 1980, on a vu naitre des SEF à la Maison d'amitié à Ottawa, à la Maison Interlude House à Hawkesbury et à celle de Kapuskasing. Ce sont des maisons d'hébergement qui offrent encore aujourd'hui des SEF en tout temps, avec quelques travailleuses en counselling dans des postes satellites multiservices.

Dès 1988, AOcVF constatait une quasi-absence de SEF dans le domaine de la violence contre les femmes, à peine quelques services offerts principalement dans la région de l'Est ontarien et, en moins grand nombre, quelques points de services dans l'immense région du Nord de l'Ontario.

La première vague de mise en œuvre de services en agression à caractère sexuel a eu lieu en 1993 et en 1994, alors que le ministère du Procureur général de l'Ontario et l'Office des affaires francophones mettaient en place, à partir d'enveloppes budgétaires totalisant $800000 \$$, un plan qui devait assurer l'accès aux SEF dans une dizaine de régions de la province. Pour la plupart, ces 
"...les organismes unilingues francophones à qui on avait confié les ressources avaient considérablement augmenté leur capacité d'offrir des services." budgets ont été confiés à des organismes existants et soi-disant bilingues.

Nous avons vite fait de conclure en l'échec de plusieurs de ces services, là où la gestion des enveloppes budgétaires des SEF avait été confiée à des organismes "dits » bilingues. En effet, en moins de cinq ans, la plupart des organismes devant offrir des SEF n'étaient plus en mesure de le faire en raison, entre autres, de l'impossibilité de recruter du personnel et de la frustration des intervenantes francophones qui devaient trop souvent offrir des services en anglais plutôt que de travailler au recrutement de la clientèle de langue française. Par ailleurs, plusieurs recherches ont démontré qu'au-delà de l'offre des meilleurs services possible, les femmes francophones se devaient d'être servies dans leur langue et que les organismes anglophones étaient incapables d'offrir aux usagères des services complets en français. Plusieurs intervenantes anglophones considéraient également qu'il s'agissait d'une injustice, voire d'une forme additionnelle de violence, à l'égard des femmes francophones et d'une non-reconnaissance de leur spécificité et de leurs besoins (Brunet et Garceau 2004).

À l'occasion de ce constat d'échec, nous avons aussi établi quelques comparaisons. Ainsi, les organismes unilingues francophones à qui on avait confié les ressources avaient considérablement augmenté leur capacité d'offrir des services. Ils avaient de plus ajouté une gamme de nouveaux services au cours de ces mêmes années, en obtenant ici et là de petites enveloppes budgétaires leur permettant d'améliorer l'accès aux services. AOcVF et ses membres ont alors recommandé la création d'entités autonomes de langue française. C’est là, pour nous, la «meilleure pratique numéro un ", essentielle à l'offre de services continus en langue française. AOcVF et ses membres ont en effet constaté qu'il existait trop de gaspillage de ressources. Par exemple, les budgets accordés aux Calacs pour les SEF étaient limités, soit entre 65000 \$ et 118000 \$, sauf pour un seul qui recevait une somme plus élevée. Il nous était donc difficile d'accepter qu'un centre bilingue n'utilise pas convenablement "son » enveloppe budgétaire de 98000 \$ pour offrir des SEF (un centre, par exemple, avait une liste de quatre usagères francophones). Nous pouvions imaginer 
aisément tout ce qui aurait pu être fait avec la même enveloppe gérée autrement. Le premier principe que nous défendons est donc l'autonomie des SEF.

\section{La meilleure pratique numéro deux : la concertation provinciale}

En 1997, sous le gouvernement Harris, une enveloppe budgétaire de 600000 \$ a été accordée aux SEF dans le domaine de la violence conjugale. Dans notre volonté d'équité pour les régions sous-desservies ou non desservies, nous avons séparé cette enveloppe en "petites pointes de tarte "! Ce montant a tout de même bien servi la mise sur pied de SEF dans le domaine de la violence contre les femmes. Nous l'avons surtout utilisé pour consolider les SEF autonomes déjà en place en ajoutant quelques programmes en violence conjugale et des postes répondant spécifiquement aux besoins des femmes immigrantes à Toronto, à Hamilton et à Ottawa.

Ces progrès sont le fruit de la concertation établie de façon régulière depuis 1992 sous l'égide d'AOcVF. Les rencontres régulières des représentantes des régions formant le conseil d'administration d'AOcVF ont permis d'établir des constats quant au développement des services, aux échecs, aux défis et aux lacunes les plus pressantes. Mais, la concertation a surtout mené à un front commun dans les représentations auprès du gouvernement provincial afin de faire valoir l'importance de la création de nouveaux services autonomes et d'obtenir le financement approprié.

Nous pouvons donc affirmer que la concertation provinciale provinciale est notre "meilleure pratique numéro deux ».» est notre "meilleure pratique numéro deux ». Elle nous a permis de travailler à obtenir de nouveaux services en français, d'améliorer ceux déjà en place et d'obtenir des projets servant des intérêts communs. Nous avons tenu des rencontres importantes, créé du matériel et formé les intervenantes.

Le démarchage politique d'AOcVF et de ses membres a permis que le principal bailleur de fonds des centres d'aide et de lutte contre les agressions sexuelles, le ministère du Procureur général, 
reconnaisse en février 2005 l'importance de l'autonomie des SEF. En plus de cela qui est déjà extraordinaire en soi, le ministère a accordé la parité de financement entre les SEF et les services en anglais dans une même région ou collectivité. Avec une nouvelle enveloppe budgétaire, il a permis la création de nouveaux centres autonomes à Thunder Bay, à Timmins, à London, dans PrescottRussell ainsi qu'un bureau satellite d'Oasis Centre des femmes à Brampton. Il a aussi ajusté le financement de centres autonomes déjà existants à Ottawa, à Toronto et à Hamilton.

Nous cherchons à obtenir le même engagement de la part du ministère des Services sociaux et communautaires (MSSC) en matière de SEF. Comme nous l'avons dit plus tôt, l'autonomie n'est pas un caprice, c'est une manière efficace de rejoindre la clientèle et de répondre aux besoins de la communauté des femmes d'expression française dans toute sa diversité : femmes immigrantes, femmes vivant avec un handicap, lesbiennes, femmes marginalisées, femmes jeunes ou âgées.

Dans l'enveloppe budgétaire annoncée l'été dernier, cette reconnaissance émerge timidement. En effet, le ministère a accordé des fonds pour des projets d'AOcVF visant le développement et la concertation, la réalisation d'études de besoins pour de nouvelles maisons d'hébergement francophones à Toronto et à Hamilton ainsi que des fonds additionnels pour la ligne francophone de soutien pour femmes victimes de violence. Des fonds sont également prévus pour de nouveaux postes d'appui transitoire et de soutien au logement, qui viendront répondre à des besoins urgents dans diverses collectivités comme l'Algoma, le comté de Renfrew, Timmins et le Sud-Ouest.

\section{La meilleure pratique numéro trois : l'adaptation des services à la réalité}

Parallèlement au démarchage pour l'obtention du financement des SEF,AOcVF et ses membres ont mis en place des modèles de services adaptés à la réalité et aux besoins des femmes francophones de la province. En voici un exemple. 
Nous venons de mentionner la ligne de soutien pour femmes victimes de violence. Permettez-nous de parler du modèle de cette ligne et d'expliquer en quoi elle répondra mieux aux besoins des femmes d'expression française qu'une ligne centralisée en un seul point de service. La ligne de soutien a été établie à partir de trois lignes qui existaient déjà dans les régions du Nord, du Sud et de l'Est. Deux d'entre elles avaient été mises sur pied par les Calacs, en partenariat avec les centres de Sudbury, de Thunder Bay et de Dubreuilville et en concertation avec Oasis Centre des femmes de Toronto et le Centre de santé communautaire de HamiltonNiagara pour leurs régions respectives. Dans l'Est, la ligne a été mise sur pied à partir de la ligne offerte par la Maison d'amitié en collaboration avec le Centre des ressources de l'Est d'Ottawa, ligne qui avait au départ une portée locale et non régionale. Lorsque le ministère de la Santé et services sociaux communautaires a offert d'améliorer les services de ligne de soutien, il a été convenu, après discussions entre les partenaires, d'utiliser un seul numéro de téléphone et de donner une identité commune aux trois lignes. Cela dans le but de faciliter la promotion du service et d'avoir une plus grande portée, c'est-à-dire, de rejoindre toutes les femmes d'expression française de l'Ontario.

"...la ligne

francophone de

soutien constitue

parfois le seul service

à la disposition d'une

femme francophone, contrairement à

l'AWH qui, elle, est un complément aux services existants, tout en offrant du soutien tant en matière de violence dans les relations intimes que dans le cas d'agressions à caractère sexuel."
Permettez-nous une parenthèse pour parler de l'offre de SEF de la ligne Assaulted Women's Helpline (AWH). À partir des services d'AT\&T basés aux États-Unis, nos consœurs de l'AWH offrent des services d'interprétation en 158 langues, dont le français. Selon nous, l'interprétation ne constitue pas un service en français. La Loi sur les services en français parle de SEF pas de services d'interprétation vers le français. Nous avons toutefois une entente de réciprocité avec l'AWH. Elles nous réferent les appels en français et nous leur transmettons les appels en anglais.

À cause des lacunes touchant les SEF et du continuum de services dans chaque région, la ligne francophone de soutien constitue parfois le seul service à la disposition d'une femme francophone, contrairement à l'AWH qui, elle, est un complément aux services existants, tout en offrant du soutien tant en matière de violence dans les relations intimes que dans le cas d'agressions à caractère sexuel. Son mandat déborde donc le simple mandat 
"La ligne ne peut pas combler les lacunes en matière de SEF, mais elle permet de mieux répondre aux besoins particuliers des femmes dans les trois régions dont les réalités sont très différentes. "

\section{"L'adaptation des} modèles de services à la réalité de la communauté de langue française constitue donc notre " troisième meilleure pratique »." de référence pour devenir un mandat de service puisque trop souvent, le service n'existe pas en français.

La ligne ne peut pas combler les lacunes en matière de SEF, mais elle permet de mieux répondre aux besoins particuliers des femmes dans les trois régions dont les réalités sont très différentes. Par exemple, on trouve très peu de femmes immigrantes dans le Nord alors qu'elles représentent un grand pourcentage des usagères à Ottawa ou à Toronto. Par ailleurs, les intervenantes qui répondent à Toronto ou à Ottawa ont parfois de la difficulté à comprendre ce que signifie l'isolement géographique que vivent les femmes dans le Nord. En présence de tant de lacunes dans la continuité des services, la ligne offre l'avantage d'être plus proche des réalités vécues par les femmes. Elle permet de maximiser les références possibles aux SEF en place dans chaque région et d'offrir un soutien immédiat aux femmes n'ayant pas accès aux services directs.

En ce qui a trait à la gestion de la ligne, il est clair que la mise en commun des ressources oblige les organismes partenaires à discuter des moindres détails, à se concerter, à mieux planifier. Mais, justement, il s'agit d'un exercice assurant une meilleure qualité des services et permettant de repérer les "points chauds » dans les besoins de l'ensemble de la province. Les partenaires ont obtenu des subventions pour une promotion commune, la formation des intervenantes et l'obtention d'un avis juridique. Il s'agit là d'une utilisation maximale des ressources disponibles. La ligne est un excellent exemple de meilleure pratique sur le plan de l'offre de services.

L'adaptation des modèles de services à la réalité de la communauté de langue française constitue donc notre « troisième meilleure pratique ».

\section{La meilleure pratique numéro quatre : une combinaison de services}

Une autre des pratiques communes aux SEF est d'avoir intégré dans les organismes et sous le même toit des services en violence dans les relations intimes et en violence à caractère sexuel, là où il était approprié de le faire. 
"Plusieurs des SEF ont à la fois un mandat local et un mandat régional. "
Dans les régions où les ressources consacrées à la mise sur pied de services étaient limitées, les centres ont choisi de réunir sous un même toit les services liés aux deux problématiques. D'une part, parce qu'il s'agissait souvent du seul SEF de la collectivité et que, par conséquent, il était appelé à répondre aux deux problématiques. D'autre part, parce qu'il était impossible de créer deux structures administratives distinctes (une maison d'hébergement et un Calacs), compte tenu du peu de ressources financières.

Cela s'est fait surtout ailleurs que dans l'Est ontarien où, en raison de la concentration importante de la population francophone, on a pu mettre sur pied des organismes autonomes pour les services en violence conjugale et en agression à caractère sexuel. Récemment, comme suite à l'annonce du ministère du Procureur général de février 2005, d'autres régions ont maintenant des services spécialisés en agression sexuelle. Cela étant dit, rappelons que dans plusieurs coins de la province, il n'existe toujours aucun SEF.

\section{La meilleure pratique numéro cinq : un mandat régional}

Plusieurs des SEF ont à la fois un mandat local et un mandat régional. C'est le cas du CentreVictoria pour femmes de Sudbury qui offre des services dans l'Algoma grâce à des bureaux satellites à Wawa, à Elliot Lake et à Sault-Ste-Marie. Situé à London, le Carrefour des femmes du Sud-Ouest a pour mandat de desservir les femmes aux prises avec la problématique de l'agression sexuelle dans toute cette région. Oasis Centre des femmes de Toronto a un bureau satellite pour desservir les femmes de Brampton. Calacs situé à Casselman, le Centre Novas desservira sous peu l'ensemble des femmes des comtés de Prescott et Russell. Les Services d'approche mis sur pied par la Maison Interlude House ont également un mandat régional et desservent les comtés de Stormont-Dundas-Glengarry et de Prescott-Russell. Ils ont été mis sur pied pour répondre aux besoins des femmes des comtés ruraux de l'Est ontarien, là où il n'y a pas de transport en commun ni, de façon générale, de moyens d'accès aux services près de 
chez soi. De plus, dans ces comtés, les femmes n'avaient pas nécessairement besoin d'avoir accès à une maison d'hébergement, mais plutôt de recevoir du soutien pour elles et leurs enfants (soutien pour obtenir l'aide sociale ou un logement, pour avoir accès à certains programmes ou à l'aide juridique, etc.). C'est donc un programme innovateur qui offre une gamme de services aux femmes et qui pourrait très bien servir de modèle dans d'autres régions.

\section{Discussion}

Nos meilleures pratiques sont très différentes des modèles existants et elles illustrent bien la possibilité d'innover afin de faire face aux nombreux défis, entre autres, celui de desservir une population dispersée de femmes francophones ayant des demandes multiples et variées auprès d'organismes qui ne disposent que de ressources très limitées.

La mise sur pied des SEF s'est faite en grande partie grâce à leur persévérance et à l'étroite collaboration entre AOcVF et ses groupes membres. Nous avons réussi à démontrer qu'il est possible de créer des SEF et qu'ils peuvent être efficaces et de bonne qualité. Nous avons pu également compter sur des alliées et alliés sûrs dans divers ministères, des personnes qui ont vraiment à cœur l'établissement de SEF en Ontario.

Il existe une dynamique propre à la communauté de langue française. Elle joue dans la création de service dans le domaine de la violence contre les femmes. Elle repose principalement sur des modèles innovateurs, une concertation qui sert bien les services existants, car elle permet d'offrir des formations communes, un soutien mutuel dans la création des services, l'échange d'outils, la capacité de déterminer où sont les lacunes et quelles sont les solutions pour les combler.

Ainsi, lorsque les gestionnaires de programmes des ministères ont à prendre des décisions au sujet des SEF, ils doivent être à l'écoute de cette dynamique fort différente. La mise sur pied de services ne fonctionne pas de la même façon dans les groupes minoritaires. Si, par exemple, on devait penser à établir une maison 
"Au cours des vingt dernières années, le tissu démographique de l'Ontario a énormément changé. En effet, la communauté de langue française de l'Ontario est passée d'une communauté pratiquement homogène de race blanche et catholique qui accueillait principalement des immigrants européens (France, Belgique, etc.) à une société multiculturelle dont les nouveaux arrivants, depuis 1996, proviennent surtout de l'Afrique du Nord et de l'Afrique subsaharienne. " d'hébergement francophone à côté de chacune des cent maisons d'hébergement anglophones déjà existantes, on n'en finirait plus! Par contre, lorsqu'on ne craint pas d'innover et de donner aux femmes d'expression française les outils dont elles ont besoin pour la création de services particuliers à leur communauté,les modèles de services sont différents, les indicateurs de rendement sont différents, la gestion est différente. Mais, chacun de ces éléments est tout aussi efficace. Il est donc possible de faire les choses autrement. Nous avons l'intention de continuer à le faire.

À Timmins, par exemple, les femmes étaient insatisfaites des services donnés par le centre " dit » bilingue en matière d'agression sexuelle. D'après ce centre, toutes les usagères étaient bilingues, il n'y avait donc pas vraiment de demande de services en français. Dès l'annonce de la mise en œuvre du service autonome de langue française, le Centre Passerelle, plus de 30 femmes ont téléphoné pour proposer leurs services comme bénévoles ou pour siéger au conseil d'administration. Le Centre Passerelle s'était fixé un objectif de 600 " contacts " dans la collectivité. Au cours de sa première année d'existence, il y en eut plus de 900 , alors que les éléments de résistance de la communauté n'avaient prédit que des scénarios de catastrophe pour Timmins et répété qu'il était inutile de mettre sur pied un nouveau service autonome géré par et pour les femmes francophones.

Nous aimerions faire un constat au sujet de l'intégration des immigrantes et des immigrants dans les SEF. Nous avons récemment discuté des changements dans la société ontarienne et de la composition de la communauté de langue française en 2007. Au cours des vingt dernières années, le tissu démographique de l'Ontario a énormément changé. En effet, la communauté de langue française de l'Ontario est passée d'une communauté pratiquement homogène de race blanche et catholique qui accueillait principalement des immigrants européens (France, Belgique, etc.) à une société multiculturelle dont les nouveaux arrivants, depuis 1996, proviennent surtout de l'Afrique du Nord et de l'Afrique subsaharienne. Lors de la vague d'immigration en provenance de l'Asie au début des années 1980, bien que plusieurs nouveaux arrivants, principalement du Vietnam et du 
"Au début des années 1980, il n'y avait pas de SEF pour accueillir ces nouveaux arrivants. Il était donc "naturel " que la majorité d'entre eux s'assimilent à la communauté majoritaire et adoptent automatiquement, ainsi que leurs progénitures, la langue de la majorité."
Cambodge, maîtrisaient le français, un nombre limité d'entre eux s'est intégré à la communauté de langue française. Il est surprenant de constater que les statistiques indiquent que le nombre de personnes en provenance des pays d'Asie qui se définissent comme francophones dépasse le nombre de personnes d'origine africaine vivant actuellement en Ontario. Pourtant, on constate qu'un plus grand nombre d'Africains et d'Africaines s'intègrent à la communauté de langue française.

Que s'est-il passé entre ces deux vagues d'immigration pour que les nouvelles arrivantes et les nouveaux arrivants s'intègrent davantage à la francophonie? Sans aucun doute la mise en œuvre de SEF, grâce en grande partie à l'adoption de la Loi sur les services en français. Au début des années 1980, il n'y avait pas de SEF pour accueillir ces nouveaux arrivants. Il était donc "naturel " que la majorité d'entre eux s'assimilent à la communauté majoritaire et adoptent automatiquement, ainsi que leurs progénitures, la langue de la majorité. Effectivement, dans plusieurs régions et dans plusieurs secteurs d'activités, les SEF n'existaient pratiquement pas et, pour recevoir des services de santé, des services sociaux ou pour s'inscrire aux études, il fallait parler anglais.

Toutefois, l'adoption d'une loi n'est pas une fin en soi. Une fois que les droits des francophones sont reconnus en Ontario, il faut non seulement concrétiser l'application stricte de la loi, mais aussi son " esprit ", sa signification en termes de reconnaissance des besoins des francophones dans l'ensemble de la province. Cette application, tant en principe qu'en pratique, devrait se traduire par un accès de plus en plus grand à des services équivalents aux services en anglais, dans tous les secteurs d'activités.

C'est là que s'inscrivent les « meilleures pratiques » d'autonomie, de concertation provinciale régulière et d'adaptation de modèles de services innovateurs et qu'elles prennent toute leur importance. Comment répondre efficacement aux besoins de la communauté francophone dans sa diversité en assurant la continuité dans les services et la pérennité dans chacune des régions? 


\section{Conclusion}

Il n'y a pas de recette miracle pour éliminer la violence contre les femmes.Au contraire, il s'agit d'un travail de longue haleine. Tenter de rejoindre toutes les femmes francophones aux prises avec la violence et leur offrir le soutien dont elles ont besoin en plus de faire de la prévention et de la sensibilisation dans les collectivités ou dans les écoles est un travail ardu. Cela veut dire siéger à de multiples comités, intervenir auprès des services policiers, du système de justice et des médias. Les tâches sont nombreuses, mais les ressources restreintes.

Lorsque le gouvernement met à la disposition des collectivités les ressources nécessaires, elles sont utilisées efficacement. Les femmes francophones offrant des services dans le domaine de la violence conjugale et de l'agression à caractère sexuel ont prouvé qu'elles peuvent le faire avec efficacité, qu'elles sont organisées, qu'elles ont les infrastructures nécessaires et qu'elles obtiennent les résultats attendus. L'augmentation graduelle des services en français en violence faite aux femmes démontre bien qu'au fil des années le réseau des organismes a innové en mettant en place des modèles alternatifs et a parcouru beaucoup de chemin en matière de parité du financement et d'autonomie des services. Ces victoires importantes ne servent cependant pas les seuls intérêts des groupes de femmes en matière de prestation de SEF. Elles ont également permis de faire avancer la cause de l'ensemble des services en français dans toutes les sphères d'activité de l'Ontario français.

\section{Bibliographie}

BRUNET, L. et M.-L. GARCEAU 2004. Faire autant avec si peu... Bilan et profil des services en français en matière de violence contre les femmes (1994-2004), Ottawa :Action ontarienne contre la violence faite aux femmes : (http://francofemmes.org/aocvf/index.cfm?Voir'activ\&\&Id'1209\&Categor ie_No'999998\&Sequence_No'1209\&niveau'2\&Repertoire_No'-1051436664

\section{Note}

Merci à Louise Allaire pour ses judicieux conseils. 